\title{
Effects of Thai dance on median neural tension, chronaxy, and nerve functions in the people associated with WMSDs.
}

\author{
Nuttanun Khemamuttanak B.Sc. (Nuttanun.khema@gmail.com) $)^{1}$, Keerin Mekhora Ph.D. \\ (Keerin.mek@mahidol.ac.th) ${ }^{1}$, Wattana Jalayondeja Ph.D. (Wattana.jal@mahidol.ac.th $)^{1}$ and \\ Sirikarn Somprasong Ph.D. (Sirikarn.som@mahidol.ac.th) ${ }^{1}$
}

${ }^{1}$ Department of Physical Therapy, the Mahidol University, Nakhon Pathom, Thailand.

\begin{abstract}
The aim of this study was to evaluate the effects of Thai dancing on the median neural tension, chronaxy, sensory response and grip strength in subjects associated with early stage work-related musculoskeletal disorders (WMSDs). Method: Thirteen computer users with early stage WMSDs voluntarily participated in the single group pre-post designed study. The neural tension of median nerve was measured via an elbow extension range of motion. Chronaxies from strength-duration curve test, the minimum time necessary to excite nerve tissue to stimulate a muscle was measured by electrical stimulator. The tested muscle was the flexor superficial digitorum muscle which was a representative muscle innervated by median nerve. The sensational response was assessed by using Semmes-Weinstein monofilament test. Muscle power from the grip strength test was also examined. All tests were performed prior to and immediately after Thai dancing (10 min). The Thai dancing program comprised of 6 basic Thai dancing movements viz. Prom See Na, Yong Fon Hang, Lor Kaew, Cha Nee Raimai, Ram Yua and Sod Soy Mala consisting of a combination of dance approximately 5-40 times for each movement, in a total of $5 \mathrm{~min}$ of dance. The participants were asked to follow the dance provided via clip video by having a first $5 \mathrm{~min}$ of practice and another $5 \mathrm{~min}$ of true dancing. Result: There were a significant decrease of neural tension $(\mathrm{p}<0.02)$ and chronaxy before and after Thai dancing $(\mathrm{p}<0.05)$ and an increase of power grip strength. While the sensory responses before and after Thai dancing were not significantly changed by using the Wilcoxon signed rank test $(p>0.05)$ The decrease of neural tension (higher degree of elbow extension) and chronaxy and the subsequent increase of power grip strength indicated an improvement of physiological response of median nerve when the neural tension was decreased. Conclusion: The subjected using the computers for a long period could develop median neural tension. This study found Thai dancing could help by reducing the neural tension and improve the nerve functions such as sensation, muscle power, and chronaxy value. Therefore, Thai dancing for $10 \mathrm{~min}$ could be beneficial for the people suffering from the early stage WMSDs especially for computer users.
\end{abstract}

Keywords: Thai dancing; median neural tension; chronaxy; nerve function; work-related musculoskeletal disorder; ESK-JES Joint Session

\section{Introduction}

Work-related musculoskeletal disorders (WMSDs) are the major health problems in many occupations and the prevalence of WMSDs is currently increasing. The symptoms of WMSDs including pain, swelling, tingling, numbness, paresthesia, stiffness, restricted range of motion and loss of strength (1-2). WMSDs caused by static poor posture, repetitive movement, use of force and prolong contraction of muscles (3) that lead to tendon, muscle, and support structure changing continuously thereby affecting the neural tension causing adverse neurodynamics or nerve function loss or nerve injury. Therefore, the relief of neural tension is extremely important (4).

The abnormal nerve tension affects the mechanical function of nerve. We can detect the mechanical function of the nervous system in the upper limb such as ULNT1 (4). Meanwhile, the abnormal nerve tension also affects chronaxy and causes loss of nerve function. The chronaxy shows one point on strength-duration 
curve. It can determine physiological change of peripheral nervous system. So using chronaxy (5) as a measurement can enhance the outcome of study. Furthermore, the loss of functions of the nerve can be detected from physical examination of sensation such as light touch or examination of motor function composed of reflex testing and muscle power test. Mekhora et al. (2015-2016) studied the effect of Thai dance on neurodynamic response in office workers by ULNT. They found that neural tension was decreased by Thai dancing. Thai dancing is a cultural art of Thailand. It uses hand movement with music which is similar to the components of standard ULNT and nerve mobilization. Therefore, this study was interested to evaluate the effect of Thai dancing on neural tension. As mentioned above, the limited number of studies were performed using nerve function as an outcome to determine the effect of Thai dancing. Therefore, the present study aimed to determine the effect of Thai dancing on median neurodynamic response, chronaxy, sensory response, and grip strength in the people associated with the early stage WMSDs.

\section{Method}

\subsection{Participants}

The present investigation was the single group pre-post designed study approved by the Mahidol University Central Institutional Review Board (MU-CIRB COA. No.2016/165.1410). The researcher announced to recruit participants for this study by signboard at the physical therapy centre of the Faculty of Physical Therapy, Mahidol University. The aims and methods of this study were explained to all participants before they signed the consent form. Thirteen volunteers ( 8 females, 3 males) with age range from 21 to 28 years. They were regular computer users having the symptoms of early stage WMSD. They must have positive ULNT1, minimal light touch impairment determined by using SWMT (size of monofilament in the range of 3.22 to 4.31 ), no numbness and no history of neck or spinal cord or brain injury, and no known history of neuropathy, neuromuscular disorders and muscle disorders. They may have WMSDs, but within early stage with pain and tiredness of the affected upper limb. This indicated that having the symptoms during work and able to get rid of it by taking rest, with no reduction of work performance. The exclusion criteria included: using drug for pain relief within 1 day before the test, limitation of neck and upper extremity motion, history of Arthritis or Impingement syndrome or Nerve irritation, having regular exercise that may have an effect on nerve tension such as Thai dancing, Yoga, Taichi, Qigong and weight training exercise, having regular treatment of physical therapy or other treatment such as massage, unable to communicate and explain feeling of pain and tension, communication disorders, moderate or severe pain at upper extremity, history of accidence at upper extremity.

\subsection{Procedures}

After the participants signed informed consents, they measured all assessments (tested in 2.3 Assessment) three times. The pretest 2 was measured after pretest 1 for one week. After that they performed Thai dancing program of $10 \mathrm{~min}$ duration by following the dance shown on the monitor. All participants performed Thai dancing under observation and care from a physical therapist. The participants were measured before $\&$ after 10 min of Thai dancing. The Thai dancing program in a total of 5 min of dance (6) (Figure 1). The participants were asked to follow the dance provided via clip video by having a first $5 \mathrm{~min}$ of practice and another $5 \mathrm{~min}$ of true dancing.

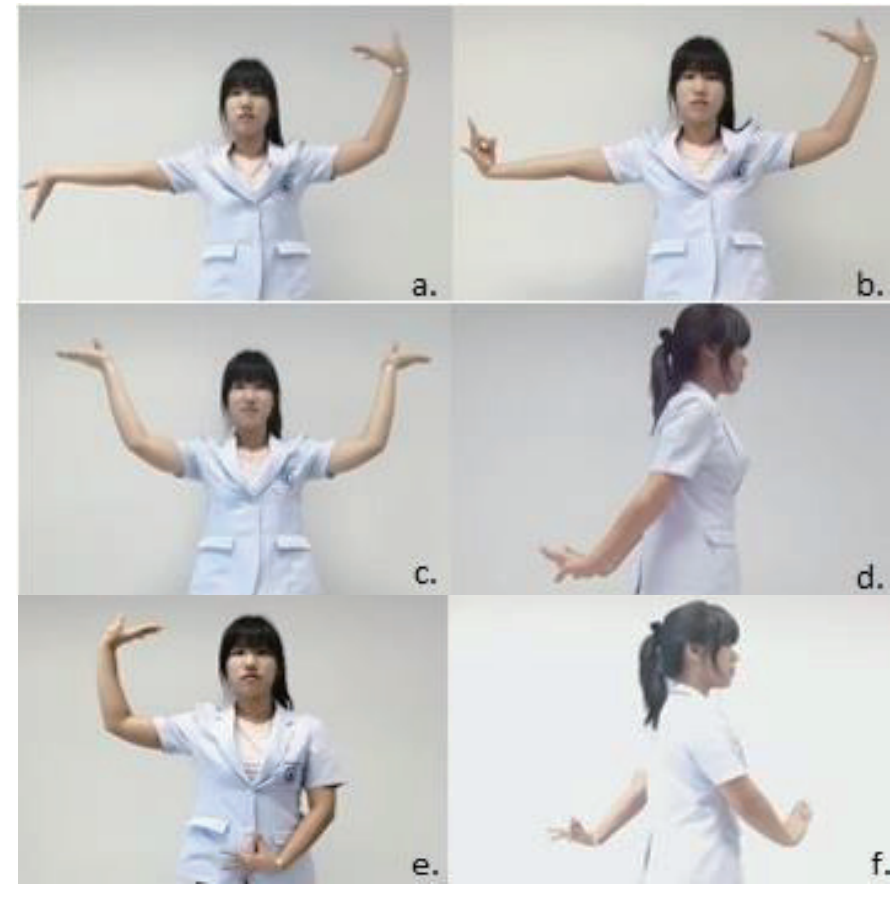

Figure 1. All performances of Thai dancing program a; Cha Nee Raimai, b; Lor Kaew， c; Prom See Na, d; Yong Fon Hang, e; Sod Soy Mala, f; Ram Yua 


\subsection{Assessment}

There were 4 assessments with the following sequences: SWMT, S-D curve test, Median neurodynamic test and Grip strength test. They were measured by the physical therapist, with intra-rater reliability (ICC $>0.9$ ).

The sensational response was assessed size of monofilament by using SWMT (7) which is a precised evaluation for sensory deficit in hand. The test can predict a quality of neural return. The filament was made from nylon. There are many sizes composed of 2.83 to 6.65 (7). The testing was performed 3 times and averaged summation of the results.

Chronaxies (ms), from strength-duration curve test, is the minimum time necessary to excite nerve tissue to stimulate a muscle by electrical stimulator. The flexor superficial digitorum muscle was a representative of the test. The flexor superficial digitorum muscle was used to locate the motorpoint and measured distances between the pisiform bone and motor point for accuracy next testing. The test was a monopolar technique. The amount of current in $\mathrm{mA}$ required to produce a minimally perceptible sensation was determined for various duration of square-wave pulses ranging from stimulate pulse 100-0.01 $\mathrm{ms}$, interval pulse $1000 \mathrm{~ms}$ and intensity 0-50 mA. Chronaxies (ms) is the least time of an effective electrical stimulus having a strength equal to double rheobase (mA) which the least strength required for excitation (Figure 2).

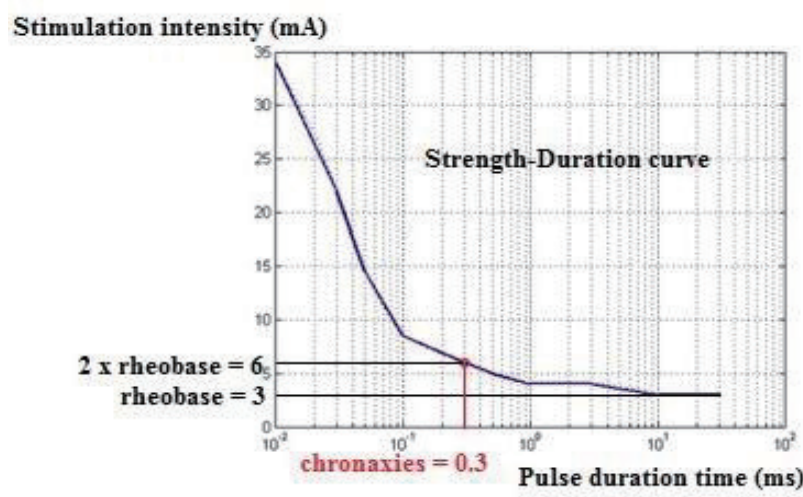

Figure 2. An example graph of one subject showed the S-D curve representation of the measurement of chronaxie.

The neural tension of median nerve measured via an elbow extension range of motion (degree) from the median neurodynamic test (8) and recorded video from a camera setting on the ceiling, perpendicular with bed. The image was then captured when light signals were pressed by participants or researcher. The participant pressed on when feeling of P1 (pain onset) and pressed again when feeling of P2 (submaximal pain limit). The researcher pressed a foot switch on when feeling of R1 (first resistance) and pressed again when feeling of R2 (end of resistance). The MATLAB program was used for calculation of angle. (Figure 3).

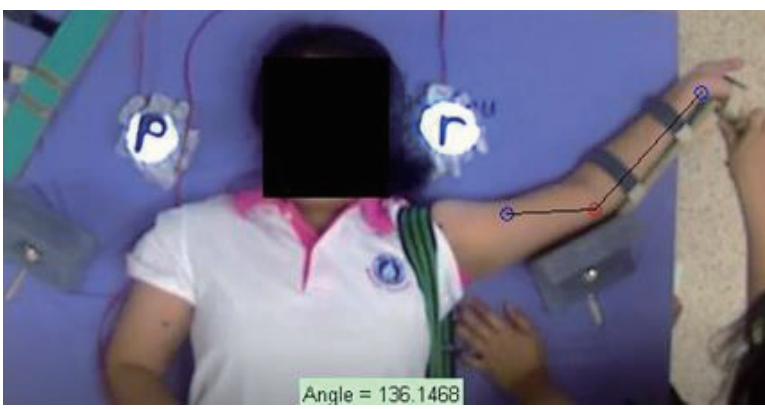

Figure 3. The captured image used MATLAB program for the measurement of an elbow extension range of motion at P1and R1.

Muscle power (kilogram) from the grip strength test was also examined. The test measured the maximum isometric strength of the combined action of forearm and hand muscle by JAMAR grip dynamometer (9). The testing on the both hands were performed 3 times, and the mean of those values were calculated. The mean score of three trails was recorded, with 1 min recovery between each effort.

\subsection{Statistical analysis}

Dependent t-test was used to determine effects of Thai dancing on median neural tension, chronaxy and grip strength. Wilcoxon signed rank test was used to determine the effects of Thai dancing on sensory response.

\section{Results}

The result between pretest 1 and pretest 2 did not showed any significant difference. The result between pretest 2 and posttest (immediately after $10 \mathrm{~min}$ of Thai dancing program) both sides showed that there were a significant decrease of chronaxies, decrease of neural tension and increase of power grip strength. While the sensory responses were not significantly changed (Table $1)$. 
Table 1. Comparison of parameters between Pretest2 and Posttest.

\begin{tabular}{|c|c|c|c|c|}
\hline \multirow{2}{*}{ Parameters } & \multirow{2}{*}{ Sides } & \multicolumn{2}{|c|}{$\mathrm{Mean} \pm \mathrm{SD}$} & \multirow{2}{*}{ P-values } \\
\hline & & Pretest 2 & Posttest & \\
\hline \multirow{2}{*}{$\begin{array}{c}\text { Sensory } \\
\text { response } \\
\text { (level) }\end{array}$} & Rt. & $3.28 \pm 0.3$ & $3.25 \pm 0.1$ & 0.317 \\
\hline & Lt. & $3.25 \pm 0.3$ & $3.25 \pm 0.1$ & 1.000 \\
\hline \multirow{2}{*}{ Chronaxy (ms) } & Rt. & $0.32 \pm 0.1$ & $0.24 \pm 0.10$ & $0.040^{*}$ \\
\hline & Lt. & $0.30 \pm 0.2$ & $0.26 \pm 0.18$ & $0.007^{* *}$ \\
\hline \multirow{8}{*}{$\begin{array}{c}\text { Median neural } \\
\text { tension } \\
\text { response } \\
\text { (degrees) } \\
(\mathrm{P} 1, \mathrm{R} 1, \mathrm{P} 2, \mathrm{R} 2)\end{array}$} & \multirow{4}{*}{ Rt. } & $138.19 \pm 9.6$ & $142.07 \pm 9.2$ & $0.009^{* *}$ \\
\hline & & $138.36 \pm 11.1$ & $142.02 \pm 10.2$ & $0.007^{* *}$ \\
\hline & & $149.94 \pm 10.6$ & $155.65 \pm 11.3$ & $0.006^{* *}$ \\
\hline & & $149.42 \pm 11.3$ & $155.53 \pm 10.6$ & $0.010^{*}$ \\
\hline & \multirow{4}{*}{ Lt. } & $136.39 \pm 9.8$ & $140.06 \pm 10.7$ & $0.007^{* *}$ \\
\hline & & $137.31 \pm 9.7$ & $140.95 \pm 10$ & $0.006^{* *}$ \\
\hline & & $148.24 \pm 13.1$ & $151.76 \pm 14.1$ & $0.007^{* *}$ \\
\hline & & $148.24 \pm 12.5$ & $151.78 \pm 14.1$ & $>0.001^{* * *}$ \\
\hline \multirow{2}{*}{$\begin{array}{l}\text { Power grip } \\
\text { strength (kg) }\end{array}$} & Rt. & $25.95 \pm 4.81$ & $27.23 \pm 5.1$ & $0.009^{* *}$ \\
\hline & Lt. & $25.37 \pm 4.7$ & $26.45 \pm 5.8$ & $0.030^{*}$ \\
\hline
\end{tabular}

Values are mean \pm SD. ${ }^{*} p<.05,{ }^{* *} p<.01,{ }^{* * *} p<.001$; Values were statistically significantly different.

\section{Discussion}

This study investigated the immediate effects of Thai dancing for $10 \mathrm{~min}$ on median neural tension responses, chronaxy, power grip strength and sensory response in people with WMSDs. The result showed that the degree of elbow extension of all neural tension responses immediately after Thai dancing for $10 \mathrm{~min}$ were increased. This was confirmed by decreasing of chronaxy values and increasing of power grip strength, which was found immediately after Thai dancing. Thus, from the results of the median neurodynamic test, strength duration test and grip strength test indicated that Thai dancing for 10 min could immediately decrease median neural tension effectively. Thai dancing is composed of gentle oscillation of maneuver, repetitive movement and slow rhythm. Due to this characteristics of the Thai dance, it allows gliding and sliding of nerve which may increase pumping blood circulation into the nerves, and bring in more nutrient and oxygen for the function of nerve (6). So, Thai dancing-10 min can improve the physiological response of median nerve as a consequence of decreased neural tension.

There are some limitations in this study. There was no control group. However, this study measured pretest twice. Age of participants was between 21 to 28 years, indicating early adulthood. Further study should determine the effect of age on the change of neurodynamic by Thai dancing. Elderly age may decline some body systems such as stiffness of joint can inhibit the gliding of the nerve (10). This study can't suggest appropriate duration time and frequency of Thai dancing program. Further studies should investigate proper duration and frequency of Thai dancing program for the best improvement for nerve.

\section{Conclusion}

People using computers for a long period may develop median neural tension. This study found Thai dancing could reduce the neural tension and improve nerve functions such as sensation, muscle power and chronaxy value. Therefore, this study suggested that Thai dancing for 10 min could help people who have suffered from the early stage WMSDs and it can be recommended as an exercise for computer users.

\section{References}

Staal J.B., DeBie R.A., Hendriks EJM. Aetiology and management of work-related upper extremity disorders., Best Pract Res Clin Rheumatol, 21(1), 33-123, 2007.

Ming Z., Zaproudina N. Computer use related upper limb musculoskeletal (ComRULM) disorders., Pathophysiology, 9, 60-155, 2003.

Ong CN, Chian SE, Jeyaratnam J, Tan KC., Musculosketal disorders among operators of visual display terminals, Scand $J$ Work Environ Health, (21), 60-64, 1995.

Butler DS., Mobilization of the nervous system, Longman Cheshire Pty Limited, Australia.1991.

C. B. Wynn Parry, "Strength-duration curves," in Electrodiagnosis and Electromyography, Baltimore. 241-271, 1971.

Mlyamoto MD. Probability of quantal transmitter release from nerve terminals: theoretical considerations in the determination of spatial variation, J Theor Biol, 289-304, 1986.

North coast page, https://www.ncmedical.com/item_1278.html (retrieved April 19, 2017).

Upiriyasakul R., Mekhora J., Jalayondeja W., Alteration of Median Neural Tension From 4 Hours of Computer Use., The Journal of Ergonomic, S44-S50, 2016.

Gill, D., Reddon, J., Renney, C., Stefanyk, W., "Hand Dynamometer: Effects of Trials and Session," Perceptual and Motor Skills 61:195-198, 1985.

Merck Manual page, http://www.merckmanuals.com/home (retrieved April 18, 2017). 